\title{
DEAR: A Device and Energy Aware Routing protocol for heterogeneous ad hoc networks
}

\author{
Arun Avudainayagam, Wenjing Lou, and Yuguang Fang* \\ Department of Electrical and Computer Engineering, University of Florida, Gainesville, FL 32611, USA \\ Received 17 October 2002; accepted 21 October 2002
}

\begin{abstract}
Energy efficient routing in ad hoc networks has been the subject of intensive study in recent years. Most work has been focused on residual battery power related cost metrics for a homogeneous ad hoc network, where all the nodes are considered identical in terms of functioning and available resources. In this paper, we propose a Device and Energy Aware Routing protocol (DEAR) for a heterogeneous wireless ad hoc network where there exist two different classes of nodes, battery-powered nodes and externally powered nodes. By embedding both the energy and the device-type awareness into the routing protocol, we force the externally powered nodes to forward more traffic and perform more routing functions than a battery-powered node. The simulation results show that DEAR protocol achieves better system lifetime when compared to the conventional energy efficient routing protocols. (C) 2003 Elsevier Science (USA). All rights reserved.
\end{abstract}

Keywords: Wireless ad hoc networks; Energy efficient routing; Minimum energy routing; Maximum lifetime routing; Power-aware routing; Device aware routing

\section{Introduction}

Ad hoc networks are dynamically formed, infrastructure-less, wireless multi-hop networks. They can be deployed anywhere without the need for any fixed infrastructure like base stations. The nodes configure themselves into a network and cooperatively maintain network connectivity. An intermediate node is used to forward packets if two nodes wishing to communicate are not within transmitting range of each other. Hence each node acts as a router and a host. The nodes discover multi-hop routes to each other by exchanging topology information in the form of control messages. These networks are typically deployed where there is no infrastructure available or reliable, and fast network establishment and self-reconfiguration are required. For example, soldiers in a battlefield can

\footnotetext{
This work was supported in part by the Office of Naval Research Young Investigator Award under grant N000140210464, the Office of Naval Research under grant N000140210554, and National Science Foundation Faculty Early Career Award under the contract ANIR 0093241.

${ }^{*}$ Corresponding author.

E-mail addresses: arun@dsp.ufl.edu (A. Avudainayagam), wjlou@ufl.edu (W. Lou), fang@ece.ufl.edu (Y. Fang).
}

have handsets to communicate with each other, or in emergency situations like earthquakes where the existing infrastructure has been destroyed, an ad hoc network can instantly be deployed to aid in disaster recovery. Sensor networks are a subclass of ad hoc networks wherein a group of sensors capable of making various measurements exchange information with each other.

Routing in ad hoc networks has been extensively studied over the past several years [1-6]. Traditional routing protocols can be broadly classified into three groups: source initiated (reactive/on demand), table driven (proactive) and hybrid. In the source initiated protocols, the routes are not computed until needed. This introduces a route-computation delay for the packets. In cases where this latency is not acceptable, table-driven protocols are used. In these protocols, routes to all destinations are pre-computed and stored as routing tables. The nodes update their routing tables periodically by exchanging routing table update packets. These initial routing protocols are only concerned with maintaining network connectivity in a highly dynamic environment and mainly deal with issues like route discovery and route maintenance. The routing decision is based more on the position of the node in the 
topology thus has the tendency to burden certain nodes with forwarding packets.

Energy conservation is an important issue in ad hoc networks as nodes are usually battery powered. Even though a node may not have any message of its own to transmit, its battery is drained when it acts as a router and forwards packets for other nodes. Unlike the microprocessor industry or the communication hardware industry, where computation capability or the line rate have been continuously improved (regularly doubled every 18 months), battery technology has been relatively unchanged for many years. The lifetime of the battery imposes a limitation on the operation hours of an ad hoc network. Research in energy-conserving protocols in ad hoc networks has dramatically increased recently. Researchers are investigating energy conservation at every layer in the traditional protocol stack, from the radio layer up to the transport layer and application layer [7-9]. Significant energy savings have been achieved at the physical layer, data link layer and network layer. Low power electronics, energy efficient modulation, and minimum transmission power level are some basic approaches adopted at the radio layer. The minimum transmitting power level is used to maintain the link connectivity thus the power efficiency is improved while the interference is reduced [10]. At data link layer, power-saving medium access control protocols are designed to coordinate the nodes to switch between sleeping and listening mode $[11,12]$. It is also noticed that in mobile power conserving protocols, the protocol layers are closely coupled. For example, the calculation of the minimum transmission power level sometimes depends on GPS-enabled routing protocols to provide the geographic positions of the nodes. The implementation of the sleeping mode in the data link layer is also affected by the routing informationexchange pattern at the network layer.

Energy aware routing (EAR) protocols have been proposed in response to the energy conservation requirement at the network layer. The energy here refers to the energy utilized by the nodes to transmit and receive packets, i.e. the energy utilized by the communication subsystem. The energy required for data processing and other auxiliary processing is not considered here because the processing energy depends on the mission of each node and is usually negligible when compared to the power required by the communication interface. An early goal of EAR was to select the best path such that the total energy consumed by the network is minimized. This problem has been addressed, as a minimum energy problem, in $[10,13,14]$. The basic approach is to minimize the average energy consumed per packet (as it traverses the network) or per unit flow. As in the case of minimum-hop routing, one serious drawback of this approach is that nodes will have a wide difference in energy consumption. Nodes on the mini- mum energy paths will quickly drain out while the other nodes remain intact. This results in an early death of some nodes.

In scenarios where the nodes need to work collaboratively, another objective of EAR is proposed: maximize the time taken by the first node/sensor to fail because it runs out of battery power. This time is known as the system lifetime [15,16]. Singh et al. [13] proposed a set of power-aware metrics based on battery power consumption at nodes. These metrics can be easily incorporated into existing routing protocols. One of the metrics, the minimum cost/packet metric, aims to maximize the lifetime of all nodes in the network. The minimum-cost routing algorithms using this metric achieved significant reduction in cost/packet over minimum-hop routing. The mean time to node failure was also increased. Chang and Tassiulas [16] proposed an energy-conserving routing protocol to maximize the system lifetime by balancing the energy consumption among the nodes in proportion to their energy reserves. Toh [17] investigated the minimum energy and maximum lifetime issues together and revealed that the two goals are not compatible. As a trade-off, he proposed a conditional max-min battery capacity routing scheme which chooses the shortest path if all nodes in all possible routes have sufficient battery capacity. When the battery capacity of some nodes fall below a predefined threshold, routes going through these nodes will be avoided, and therefore the time until the first node power-down is extended. These proposed schemes embed the energy awareness (each node is aware of its existing battery reserves) into the routing protocol and were proposed for a homogenous ad hoc network, where all the nodes are treated identical in terms of available resources and functioning roles. In addition, those schemes are more suitable for static networks because the benefits come from the even distribution of traffic among different nodes. While the nodes are moving independently, the savings provided by these algorithms, if any, is negligible because of the difficulty of real-time reconfiguration.

We noticed that in practical ad hoc networks, there exist two different classes of nodes, externally powered nodes and the battery-powered nodes. For example, in a battlefield or a disaster recovery scenario, some soldiers/ rescue workers may be in a mobile vehicle with an alternator on it. These vehicles may be equipped with battery chargers into which the nodes can be plugged. In such a scenario, these nodes are not limited in power in the sense that their power reserves are very large compared to the battery powers of the other nodes. This fact has been scarcely exploited by other researchers in energy-conserving protocol designs [19]. In this paper we propose a Device and Energy Aware Routing (DEAR) protocol for such a heterogeneous ad hoc network, where there exist different types of nodes. Besides the energy awareness, the proposed DEAR 
protocol also embeds additional information about the node type. We will show that, with both energy and device awareness, the system lifetime can be further increased by taking advantage of the extra capability and resources of externally powered nodes while at the same time balancing traffic among the battery-powered nodes. This lifetime increase is achievable even when the nodes move independently with respect to each other.

This paper is organized as follows: In Section 2 we first study the performance of the energy aware routing protocol and compare its performance with a few conventional ad hoc routing protocols. In Section 3 we introduce the device awareness in DEAR protocol, describe the design and operation of the routing protocol, and compare its performance with the protocol studied in Section 2. The paper is concluded in Section 4.

\section{Energy aware routing protocol}

\subsection{Maximum system lifetime routing metric}

At the network layer, power efficient routing protocols are designed to select the best path such that the total energy consumed is minimized or the system lifetime is maximized. The shortest path algorithms are still used but with other carefully designed power-aware cost metrics instead of the simple hop count metric. Power-aware metrics for determining routes with various objectives have been proposed in [13]. One metric that aims to maximize the life of all nodes in the network is defined as follows:

$c_{j}=\sum_{i=1}^{k-1} f_{i}\left(x_{i}\right)$

where $c_{j}$ is the cost of sending packet $j$ from node $n_{1}$ to node $n_{k}$ via intermediate nodes $n_{2} \ldots n_{k-1}, x_{i}$ represents the total energy expended by node $i$ so far and $f_{i}\left(x_{i}\right)$ denotes the cost or weight of node $i$. Since $f_{i}$ represents a node's reluctance to forward packets, we have chosen

$f_{i}\left(x_{i}\right)=\frac{1}{E_{i}-x_{i}}$,

where $f_{i}\left(x_{i}\right)$ denotes the current cost of using node $i, x_{i}$ denotes the energy expended by node $i$ so far (noting $x_{i}$ is time-varying), and $E_{i}$ is the initial energy of node $i$ when the network is deployed. Thus, $f_{i}$ is the reciprocal of the residual energy of node $i$. Therefore, as the energy of a node decreases the cost of using that node increases.

The authors in [13] presented some results using this metric in a shortest path routing algorithm. However, they did not implement it as a protocol. Following their lead, we incorporate this metric as the link cost function into the Distributed Bellman-Ford (DBF) routing protocol for ad hoc networks. This implementation will be referred to as the energy aware routing (EAR) protocol for the rest of this paper (not to be confused with the eavesdrop and register concept in sensor networks). The benefit of EAR protocol comes from the dynamic load balancing among different nodes. The protocol needs to keep track of the changing link costs in a timely fashion. A table-driven protocol could gather this information actively while a source-initiated routing protocol presents a large latency in obtaining such changes. This is the reason for implementing the poweraware metric in the DBF protocol (the simplest of all table-driven routing protocols). In order to reduce the routing overhead generated by the table-driven protocol, our implementation of DBF just sends periodic updates of the routing table and does not trigger an update whenever there is a change in the routing table.

\subsection{Performance of EAR}

Whenever multiple paths exist from a source to a destination, EAR makes use of different paths and tends to balance the load by optimizing the residual energy. Hence, the system lifetime will be more than that obtained by using conventional routing protocols. We evaluate its performance and compare it with conventional ad hoc routing protocols by simulation. All the simulations are implemented within the GloMoSim library [20], which is a scalable simulation environment for wireless network systems. The traffic pattern is CBR traffic with each packet 512 bytes long. The packet rate between each source destination pair (a session) is generated randomly from an exponential distribution with mean equal to one packet per second. The number of nodes and the number of sessions vary in each simulation set. However, we made the configuration of each simulation identical when comparing with different routing protocols. In our simulation, we consider the energy consumed by communication subsystems only, as almost all the EAR protocols do. Each node was initialized with a fixed amount of energy/battery reserve (20000 energy units) before network deployment. We assume that the cost for a node to send or receive a packet can be modeled as the linear addition of the following two parts, a fixed cost associated with channel access cost and an incremental cost proportional to the size of packet [21]. Since we use data packets of fixed size, we further simplify the energy consumption model as that it takes twice the energy to transmit a packet when compared to receiving a packet [11]. Two units of energy were subtracted from a nodes battery reserve for each packet transmission and one unit of energy was subtracted for every packet received at MAC layer. The time from the time of network deployment to the time of the first node failure was noted as the system lifetime. Each node had a transmission range of $1 \mathrm{k}$ and the 


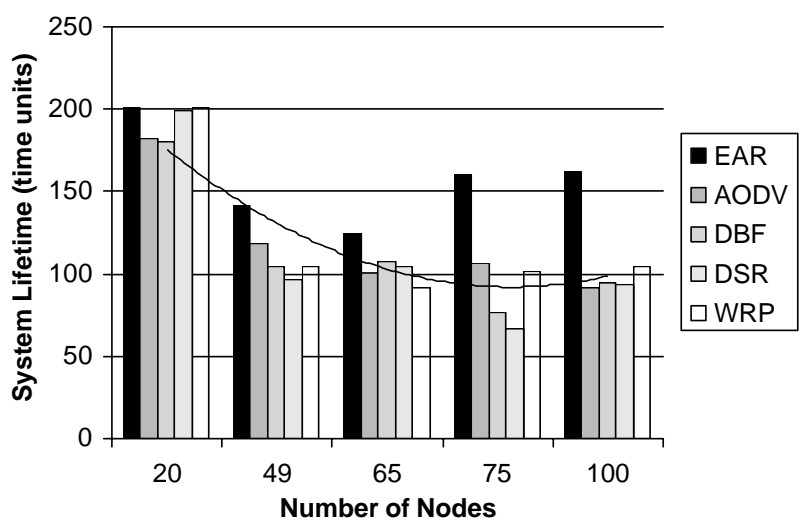

Fig. 1. System lifetime as a function of the number of nodes.

nodes were distributed over a rectangular region with minimum area of $N^{2} \mathrm{k}$, where $N$ is the number of nodes.

\subsubsection{Effect of number of nodes}

The effect of the number of nodes $N$ on the system lifetime is studied in the first simulation set. For each $N$, nodes are placed randomly with the only constraint that there should be at least a few pairs of nodes with multiple paths between them. This is because whenever there exists only a single path between pairs of nodes, the minimum-hop path and the minimum-cost (energy) path will be identical. Therefore, the performance of EAR will be the same as that of DBF. The system lifetime for a particular $N$ was obtained as the average over a number of simulations with different topologies. The results of the simulation on five different routing protocols are shown in Fig. 1. It is observed that the system lifetime is the highest when EAR is used. It can also be inferred from the trend-line shown in the figure that the system lifetime decreases with an increase in the number of nodes. This is because the number of messages that a node might forward as a router increases with an increase in the number of nodes leading to a faster depletion of energy reserves.

As the number of nodes increases, it is expected that the percentage increase in system lifetime between EAR and DBF will increase. This is expected because as the number of nodes increases, each node may have more routes (to every other node) to choose from. However, this statement cannot be generalized because the area over which the network is deployed is another parameter that affects the system lifetime. If this area is kept constant, as the number of nodes increases, nodes become crowded and many of them are within transmission range of each other, i.e. the connectivity of the network increases. Therefore, in this case, as the number of nodes increases, each node will be used as a router less frequently. Since there are more single-hop transmissions from a source node to a destination node, the performance of EAR and DBF will start converging, and the percentage increase of the system lifetime

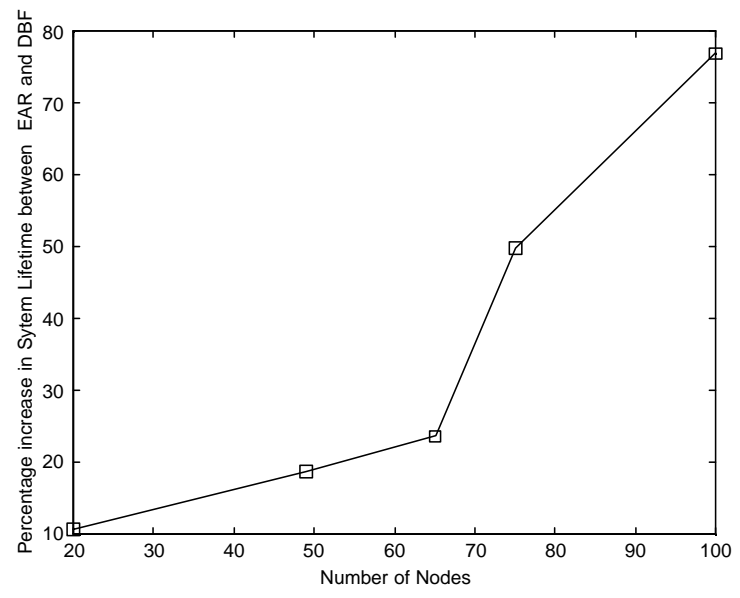

Fig. 2. Percentage increase in system lifetime between EAR and DBF.

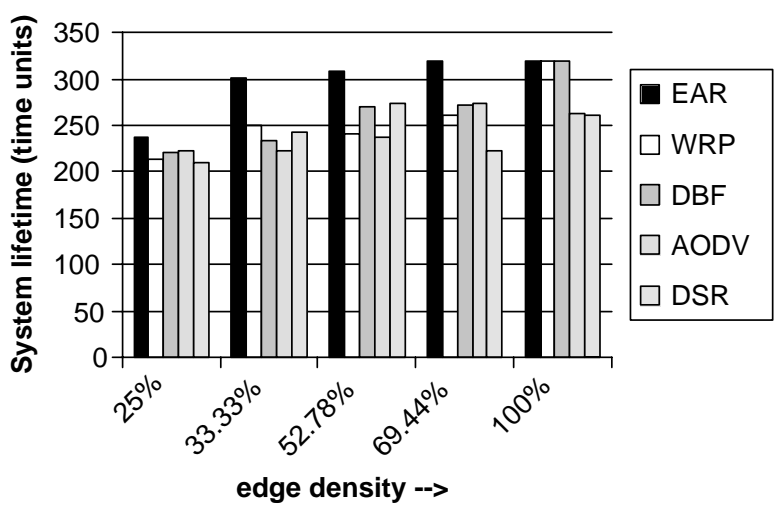

Fig. 3. System lifetime as a function of edge density.

between EAR and DBF will actually decrease with an increase in the number of nodes. This is further explained in the next section. However, in our simulation, we examine the situation where the area over which the network is deployed is also increased proportional to the number of nodes increased in the system, e.g. we keep the node density constant. As shown in Fig. 2, in this case, the percentage increase in system time with respect to DBF also increases with the increasing of the number of nodes.

\subsubsection{Effect of edge density}

This simulation set studies the effect of edge density on system lifetime. Any two nodes that can transmit and receive messages directly without the use of an intermediate node are said to have an edge between them. The ratio of the number of edges in the network and the number of edges in a fully connected network with the same number of nodes is known as edge density. The results are shown in Fig. 3. It is observed that EAR again gives the highest system lifetime. The system lifetime increases as the edge density increases since the load is balanced over a larger number of routes. 
As edge density increases, it is logical to think that the saving in cost is more since there are more routes from one node to another. Hence, it is expected that percentage increase in system lifetime between EAR and DBF will increase with an increase in edge density. This is true for wired networks but not for wireless networks. For wired networks, as the edge density increases, the connectivity of the network increases and hence there are more routes between different pairs of nodes. However, in the case of wireless networks, the only way of increasing the network connectivity keeping the number of nodes constant is by bringing more nodes within transmitting range of each other. Initially, when the edge density increases, the number of paths between pairs of nodes increases. However, after a certain edge density is reached, most of the nodes are within range of each other and messages between nodes are directly passed to one another without using an intermediate node. Hence, after the edge density threshold has been reached, nodes are used less frequently as routers. Hence the percentage increase in system lifetime starts decreasing since the shortest path starts converging to the minimum-hop path. At $100 \%$ connectivity $($ edge density $=1)$, all nodes are within range of one another and hence the network reduces to a single-hop network from a multi-hop network. Therefore, no node is used as a router and all the messages are delivered directly to the respective destinations. In this case, the shortest cost route is identical to the minimum-hop route. The percentage increase in system lifetime is zero at $100 \%$ connectivity and is not the maximum as expected. This variation in percentage increase in system lifetime is shown in Fig. 4. We also observe in Fig. 3 that EAR and DBF have the same system lifetime at $100 \%$ connectivity.

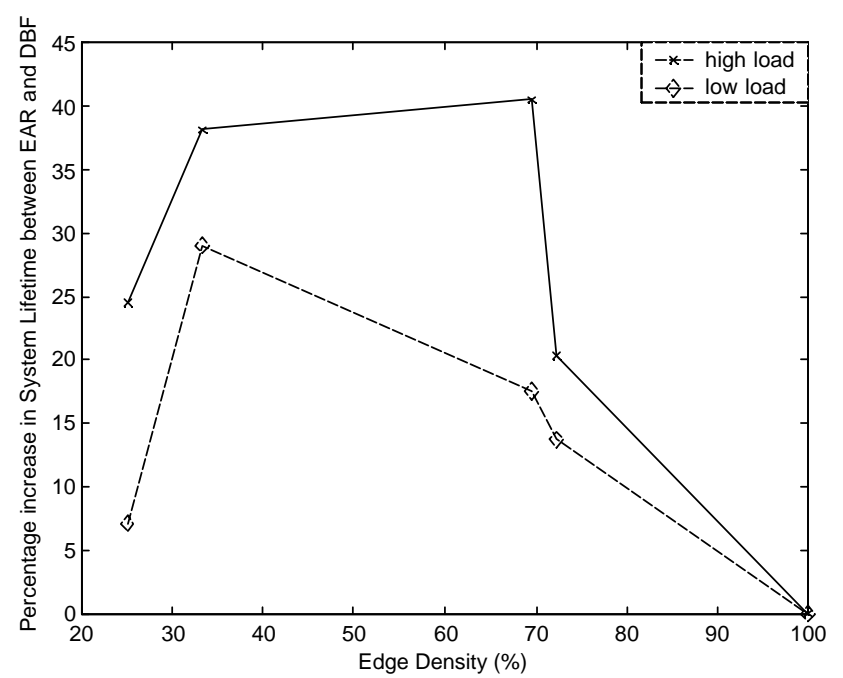

Fig. 4. Percentage increase in system lifetime between EAR and DBF as a function of edge density.
Fig. 4 also shows the percentage increase in system lifetime for two different values of load. The solid curve is the result of having a load twice that of the dashed curve. At a greater load, the percentage savings obtained by using EAR is expected to be higher than at a lower load. This is because the cost differential between paths is greater at higher load than at a lower load and hence alternate routes are used more often.

We have not considered mobility of the nodes so far. All the above results hold true only for a static ad hoc network (a sensor network kind of scenario). In [13], the authors have noticed that when the nodes move independently with respect to one another, then the savings obtained by using energy aware metrics will be small or even zero. This is because when the nodes are mobile, it is not necessary that there will be multiple paths from a source to a destination at all times. In addition, ad hoc on-demand routing protocols are not good at discovering the multiple paths in real time in response to the frequently changing link costs. However, the power aware metric will provide saving when the nodes move in a correlated way (a platoon of soldiers in a battlefield) [13]. In other applications, especially if ad hoc networks are to be pushed towards the commercial sector, this restriction on mobility is unacceptable. In the next section, we present our DEAR protocol, which provides more energy savings than pure EAR and is much less dependent on the availability of the multiple paths.

\section{The DEAR protocol}

\subsection{Motivation}

In conventional ad hoc network literature, the devices/nodes have been assumed to be identical in terms of available resources and the roles they played as a router. However, recently there has been a paradigm shift. Several researchers investigate heterogeneous ad hoc networks with the nodes not necessarily identical to each other [19]. We observe that in practical ad hoc network deployment there are some nodes that are powered by a source that is not critically limited in energy. For example, consider the deployment of an ad hoc network in a disaster situation, where the handsets carried by the rescue workers are the nodes. Then, there might be a scenario where a rescue worker plugs his handset in a charger in an automobile/ambulance. In a military application, some soldiers may be in jeeps or tanks that have chargers in them. In a commercial deployment, a user may be in a car equipped with a charger. In such scenarios, these devices are not limited in power in the sense that their power reserves are very large compared to the battery power of other nodes. The DEAR protocol that we propose exploits this fact and makes use of device awareness to enhance the routing. 


\subsection{Design and operation of DEAR protocol}

DEAR stands for Device and Energy Aware Routing. A node is said to be device aware if it can distinguish between two states: it is powered by its internal battery or it is powered by an external source. We assume that the cost of using a node powered by an external source as zero. Thus we incorporate a redirect scheme in DEAR that actively redirects the packets to the powered nodes for power-saving operations. We also assume that an externally powered node has the capability to increase its transmission power to a higher level so that it can reach any other node in the network in one hop.

The device-aware redirect scheme is designed as follows. A conventional routing table entry should include at least the following fields: destinationAddr, cost, nextHop. An additional binary field, deviceType, is added to this structure for device awareness. A 0 indicates the node destinationAddr running on its own battery and a 1 indicates the node externally powered. Each node maintains a routing table and an additional redirect table. The redirect table entry has the following structure: destinationAddr, redirectToAddr, indicating where (redirectToAdde) the packet leaving the current node to destination destinationAddr should be redirected. A -1 in field redirectToAddr indicates no redirection is needed. Whenever a routing table update is received, it updates its routing table as done in EAR or DBF. After updating its routing table, the node browses through its routing table and determines the minimum cost to reach any externally powered device. Let $C$ represent this cost and let $P$ be the id of the corresponding device. For each entry in the redirect table, if the cost to that destination is bigger than $\mathrm{C}$, packets to that destination will be redirected to $\mathrm{P}$, correspondingly the field redirectToAddr will be set to P. Otherwise, the field redirectToAddr will be set to -1 , indicating that packets to that destination should follow the routing table and not be redirected. The algorithms used to update the routing table and the redirect table are summarized in Fig. 5.

Whenever a node receives a packet to be forwarded, it extracts the destination address from the header and looks at the corresponding entry in the redirect table. If the entry in the redirect table is -1 , the node just forwards the packet to the nextHop according to the routing table for that particular destination. If the entry in the redirect table is the id of some other node, it redirects the packet to that particular node.

Once a powered node receives a packet, it checks if the destination of the packet is one of its neighbors (single-hop nodes). If so, the node unicasts it to that particular destination. If not, it boosts its transmit power to cover the entire network and unicasts the packet to its destination. So it is just a single hop from a powered node to the destination. Since the node is
TABLE UPDATE ALGORITHM

on receiving routing table update from neighbor: UpdateRoutingTable( ); /*identical to DBF update procedure*/

/*find the least cost powered node and the cost ${ }^{*}$ /

ShortestCostToPoweredNode=infinite;

for each entry $d$ in the routing table (RT), do \{

if $\left(\mathrm{RT}[\mathrm{d}]\right.$.deviceType $==1 /{ }^{*}$ device is powered node $*$

AND

RT[d].cost < ShortestCostToPoweredNode) \{ ShortestCostToPoweredNode = RT[d].cost;

\} RedirectNode $=\mathrm{RT}[\mathrm{d}]$.destinationAddr;

\}

/*Update redirect table*/

for each entry $d$ in routing table(RT) and redirect table(RD), do \{

if (RT[d].cost > ShortestCostToPoweredNode)

$\mathrm{RD}[\mathrm{d}]$. redirectToAddr $=$ RedirectNode; else

$\mathrm{RD}[\mathrm{d}]$. redirectToAddr $=-1$; \}

Fig. 5. Routing and redirect table update algorithm.

externally powered it can boost its transmit power to cover any distance. The amount by which the power has to be increased can be handled in different ways. If the area of deployment is known then the amount by which the transmit power should be increased to cover the entire area can be pre-programmed into the node. Else, if the nodes also exchange hop count information (as in DBF) apart from the cost information, then the externally powered node will know the number of hops to the intended destination and can correspondingly increase the transmit power. If the nodes had different transmit powers, DEAR operation could be combined with a protocol that exchanges transmit power information like PARO [18] to exactly determine the amount of transmit power required to reach the destination. This, however, would involve a little more over-head. For our simulations, we pre-programmed the increase in transmit power required to cover the area of network deployment into the protocol. In practice, even the powered node has a limitation in transmitted power, therefore, a powered node can increase the transmit power level to the maximum or until another powered node is found. This approach will be investigated in the future. Fig. 6 is an illustration of DEAR operation compared with EAR and minimum-hop routing.

\subsection{Performance}

Since the cost of reaching a powered node is lower, a savings in energy (a number of hops are avoided) is obtained. Fig. 7 shows the percentage improvement in 


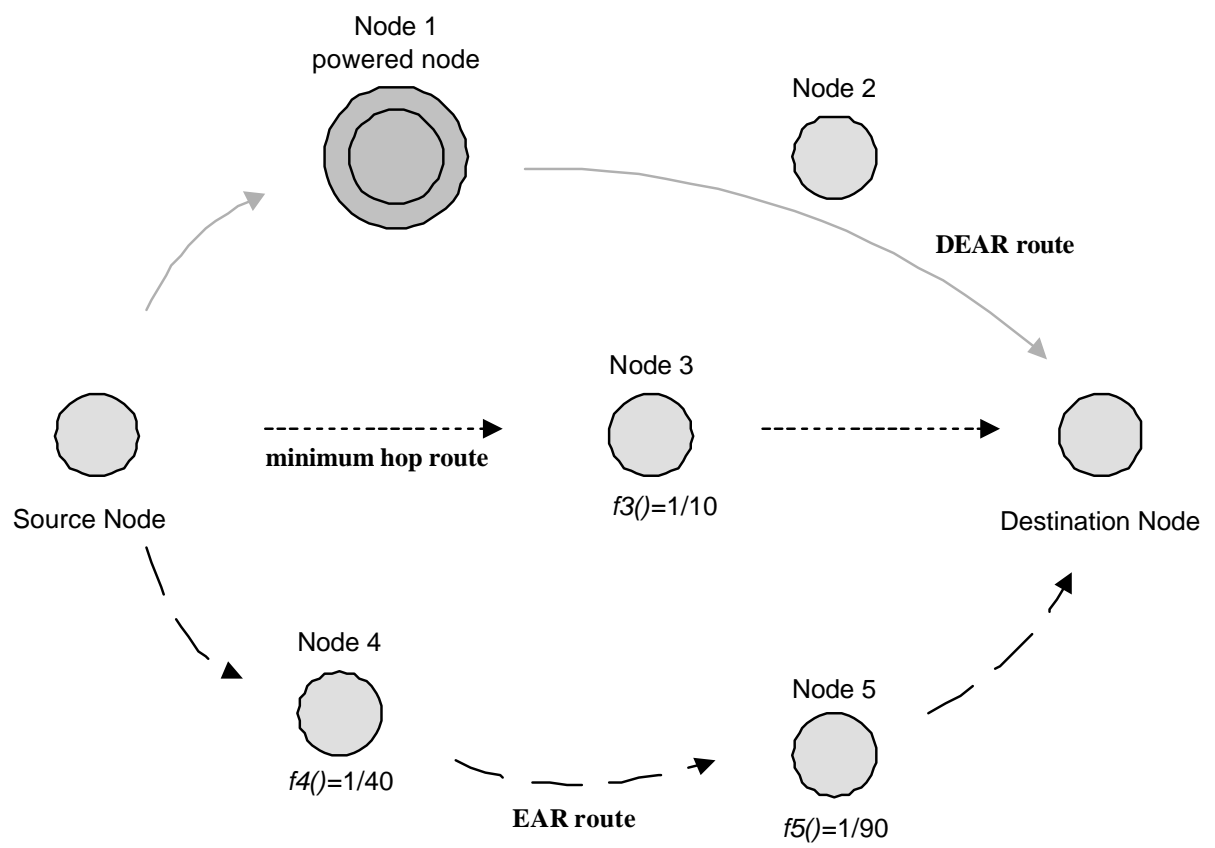

Fig. 6. An illustration of DEAR operation.

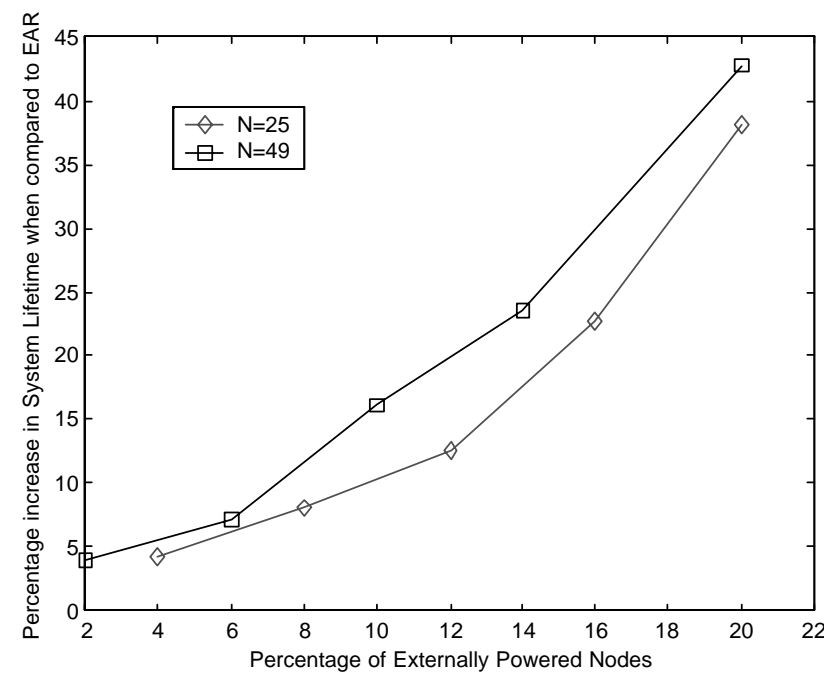

Fig. 7. Percentage increase in system lifetime between DEAR and EAR as a function of the number of nodes.

system lifetime between DEAR and EAR as a function of the number of powered nodes. It is observed that the system lifetime increases as the number of powered nodes increases, which is expected. We have not plotted the improvement in system lifetime for more than $20 \%$ of the nodes being externally powered because that is a very unlikely event. However, we would like to state that when all the nodes are externally powered $(100 \%$ powered nodes), the system lifetime is infinity as none of the nodes fail. For the same percentage of powered nodes, we observe that the increase in system lifetime is more when the total number of nodes is more. This is obvious since the number of powered nodes is greater in this case.

As in Section 2, the above simulation is run with static nodes. However, the real advantage of DEAR is not apparent in the static case. We noted, while concluding Section 2, that when nodes move independently with respect to one another, EAR does not have any apparent advantage due to the lack of multiple routes. However, when we use DEAR, even if there is a single route from source to a destination, if there existed a shorter route to a powered device (for example, a powered device happened to lie on the route between the source and the destination), then DEAR provides savings by eliminating a number of hops. The increase in system lifetime by using DEAR instead of EAR in a mobile environment is shown in Fig. 7. Nodes (25) with the random waypoint mobility model developed by Johnson and Maltz [22] were used for our simulation. The nodes move around with speeds uniformly distributed between 0 and $5 \mathrm{~m} / \mathrm{s}$. The pause time set to $0 \mathrm{~m} / \mathrm{s}$ to simulate continuous motion. If any savings is to be expected from EAR, there should be multiple routes between pairs of nodes. In a mobile environment, the only way to ensure multiple routes is by reducing the area over which the nodes move. While this only makes it more likely for EAR to provide savings, it does not guarantee better performance. This is because when the area of deployment is reduced, it is likely that the connectivity increases and as mentioned in Section 2, savings might decrease. However, in applications of mobile ad hoc networks, this constraint on the area of deployment is not practical. Hence as the area increases, the savings obtained by EAR if any, will actually 


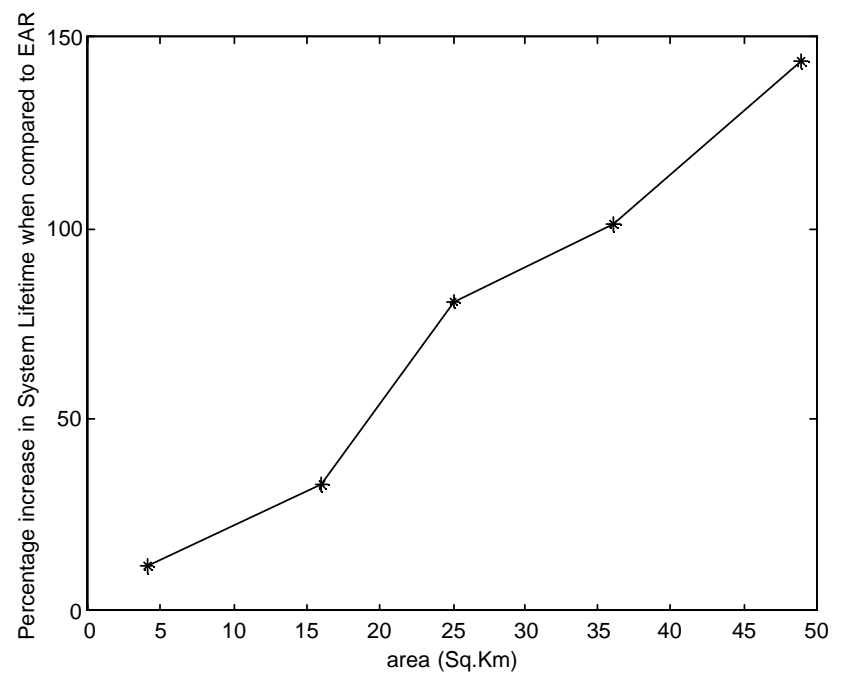

Fig. 8. Percentage increase in system lifetime as a function of the area of deployment.

decrease. It is also expected that as the area increases the number of hops between two nodes will increase. However, if we use DEAR, as the area increases, if a route exists with a smaller cost to a powered device, then the savings will be greater because it is more likely that we will avoid a greater number of hops. Hence with an increase in area, the percentage increase in system lifetime when compared to EAR should actually increase which is observed in Fig. 8.

$A$ note on the MAC layer issues: In our simulations a modified version of the MACA [23] protocol was used in the MAC layer. Whenever a powered node has to boost the transmit power and deliver a message, it transmits a special RTS packet on a separate control channel. Any node receiving the special RTS packet stops its transmissions, if any. This prevents collisions with parts of the ad hoc network that are not "visible" to the powered node. This pre-emptive MAC protocol is not optimal and better protocols will be investigated in the future. However, for low bit rate applications, the penalty of using this MAC protocol may not be severe.

\section{Conclusions}

In this paper, we compare the performance of a simple energy-aware routing protocol with a few conventional protocols using the system lifetime as the performance measure. A limitation of the application of this protocol to mobile networks is identified. The crux of our paper is not to propose better energy aware metrics but to show how these metrics can be adapted to mobile ad hoc networks. We introduce the DEAR protocol and show how embedding some device awareness into a traditional energy aware routing protocol could be used to significantly increase the system lifetime and could be used to adapt energy aware routing protocols into the mobile environment. This improvement comes with an insignificant increase of one bit per routing table entry in the routing overhead. We would like to state that device awareness could be embedded in any protocol at any layer to improve the system lifetime.

\section{Acknowledgments}

We would like to thank Mr. Nandkishore Muralidharan, Department of Electrical and Computer Engineering, for helping us in identifying some bugs in our simulation and helping us correct it.

\section{References}

[1] C.E. Perkins, P. Bhagwat, Highly dynamic destination-sequenced distance-vector routing (DSDV) for mobile computers, Comput. Commun. Rev. 24 (4) (October 1994) 234-244.

[2] S. Murphy, J.J. Garcia-Luna-Aceves, An efficient routing protocol for wireless networks, ACM Mobile Networks App. J. (Special Issue on Routing in Mobile Communication Networks) 1 (2) (1996) 183-197.

[3] D.B. Johnson, D.A. Maltz, Y.-C. Hu, J.G. Jetcheva, The dynamic source routing protocol for mobile ad hoc networks, IETF Internet Draft, draft-ietf-manet-dsr-06.txt, November 2001.

[4] C.E. Perkins, E.M. Belding-Royer, S.R. Das, Ad hoc on-demand distance vector (AODV) routing, IETF Internet draft, draft-ietfmanet-aodv-09.txt, November 2001.

[5] E.M. Royer, C.-K. Toh, A review of current routing protocols for ad hoc mobile wireless networks, IEEE Pers. Commun. 6 (2) (April 1999) 46-55.

[6] J. Broch, D. Maltz, D. Johnson, Y.-C. Hu, J. Jetcheva, A performance comparison of multi-hop wireless ad hoc network routing protocol, The Fourth Annual ACM/IEEE International Conference on Mobile Computing and Networking (MobiCom), Dallas, TX, October 1998, pp. 85-97.

[7] L. Bononi, M. Conti, L. Donatiello, A distributed mechanism for power saving in IEEE 802.11 wireless LANs, Mobile Networks Appl. 6 (3) (June 2001) 211-222.

[8] K. Sohrabi, J. Gao, V. Ailawadhi, G.J. Pottie, Protocols for selforganization of a wireless sensor network, IEEE Pers. Commun. 7 (5) (October 2000) 16-27.

[9] M. Zorzi, R.R. Rao, Energy efficiency of TCP in a local wireless environment, Mobile Networks Appl. 6 (3) (2001) 1333-1344.

[10] V. Rodoplu, T.H. Meng, Minimum energy mobile wireless networks, IEEE J. Selected Areas Commun. 17 (8) (1999) 265-278.

[11] S. Singh, C.S. Raghavendra, PAMAS-power aware multi-access protocol with signaling for ad hoc networks, ACM Comput. Commun. Rev. 28 (3) (July 1998) 5-26.

[12] B. Chen, K. Jamieson, H. Balakrishnan, R. Morris, SPAN: an energy efficient coordination algorithm for topology maintenance in ad hoc wireless networks, ACM Mobicom'01.

[13] S. Singh, M. Woo, C.S. Raghavendra, Power aware routing in mobile ad hoc networks, Proceedings of the Fourth Annual ACM/IEEE International Conference on Mobile Computing and Networking (MobiCom '98), Dallas, TX, October 1998, pp. 181-190. 
[14] X. Li, P. Wan, Constructing minimum energy mobile wireless networks, ACM Mobile Comput. Commun. Rev. 5 (4) (2001) $55-67$.

[15] W. Heinzelman, A. Chandrasekaran, H. Balakrishnan, Energy efficient communication protocol for wireless micro sensor networks, Proceedings of the 33rd Hawaii International Conference on System Sciences (HICSS '00), Big Island, HI, January 2000, pp. 3005-3014.

[16] J. Chang, L. Tassiulas, Energy conserving routing in wireless ad hoc networks, Proceedings IEEE INFOCOM 2000, Tel-Aviv, Israel, March 2000, pp. 22-31.

[17] C.-K. Toh, Maximum battery life routing to support ubiquitous mobile computing in wireless ad hoc networks, IEEE Commun. Mag. 39 (6) (2001) 138-147.

[18] J. Gomez, A.T. Campbell, M. Naghshineh, C. Bisdikian, Poweraware routing in wireless packet networks, Proceedings of Sixth IEEE International Workshop on Mobile Multimedia Communications, San Diego, CA, November 1999.

[19] J.-H. Ryu, D.-H. Cho, A new routing scheme concerning energy conservation in wireless home ad-hoc networks, IEEE Trans. Consumer Electron. 47 (1) (2001) 1-5.

[20] M. Takai, L. Bajaj, R. Ahuja, R. Bagrodia, M. Gerla, GloMoSim: a scalable network simulation environment, Technical Report 990027, UCLA, Computer Science Department, 1999.

[21] L.M. Feeney, An energy consumption model for performance analysis of routing protocols for mobile ad hoc networks, Mobile Networks Appl. 6 (2001) 239-249.

[22] D.B. Johnson, D.A. Maltz, Dynamic source routing in ad hoc wireless networks, in: T. Imielinski, H. Korth (Eds.), Mobile Computing, Kluwer Academic Publishers, Dordrecht, 1996, pp. 153-181 (chapter 5).

[23] P. Karn, MACA - a new channel access method for packet radio, in: ARRL/CRRL Amateur Radio Nineth Computer Networking Conference, ARRL, 1990, pp. 134-140.

Arun Avudainayagam received the B.E degree in Electronics and Communication Engineering from Anna University, India in 2000. In 2001, he received the M.S degree in Electrical and Computer Engineering from the University of Florida where he is currently pursuing his Ph.D degree. His research interests include wireless ad hoc networks, error correction coding and iterative decoding techniques.

Wenjing Lou received the B.E degree and M.E degree in Computer Science and Engineering from Xi'an Jiaotong University, China, in
1993 and 1996 respectively. She received the M.A.Sc degree in Computer Communications from Nanyang Technological University, Singapore, in 1998. From Dec 1997 to Jul 1999, she worked as a Research Engineer in Network Technology Research Center, Nanyang Technological University. She is currently pursuing the $\mathrm{Ph} . \mathrm{D}$ degree in Electrical and Computer Engineering at University of Florida. Her research interests include wireless ad hoc networks, routing protocols in both wired and wireless networks, and network security. Wenjing Lou is a member of Tau Beta Pi and a student member of the IEEE.

Dr. Fang received a Ph.D degree in Systems and Control Engineering from Case Western Reserve University in January 1994, and a Ph.D degree in Electrical Engineering from Boston University in May 1997. From June 1997 to July 1998, he was a Visiting Assistant Professor in Department of Electrical Engineering at the University of Texas at Dallas. From July 1998 to May 2000, he was an Assistant Professor in the Department of Electrical and Computer Engineering at New Jersey Institute of Technology. Since May 2000, he has been an Assistant Professor in the Department of Electrical and Computer Engineering at University of Florida. His research interests span many areas including wireless networks, mobile computing, mobile communications, automatic control, and neural networks. He has published over sixty papers in refereed professional journals and conferences. He has received the National Science Foundation Faculty Early Career Development Award in 2001 and the Office of Naval Research Young Investigator Award in 2002. He is listed in Marquis Who's Who in Science and Engineering, Who's Who in America and Who's Who in World.

Dr. Fang has engaged in many professional activities. He is a senior member of the IEEE and a member of the ACM. He is an Editor for IEEE Transactions on Communications, an Editor for IEEE Transactions on Wireless Communications, an Editor for ACM Wireless Networks, an Area Editor for ACM Mobile Computing and Communications Review, an Associate Editor for Wiley International Journal on Wireless Communications and Mobile Computing, and Feature Editor for Scanning the Literature in IEEE Personal Communications. He has also actively involved with many professional conferences such as ACM MobiCom'02 (Co-Chair for Student Travel Award Committee), ACM MobiCom'01, IEEE INFOCOM'03, INFOCOM'00, INFOCOM'98, IEEE WCNC'02, WCNC'00 (Technical Program Vice-Chair), WCNC'99, and International Conference on Computer Communications and Networking (IC3N'98) (Technical Program Vice-Chair). 